\title{
OPEN School-based screening and treatment may reduce $P$. falciparum transmission
}

\author{
Lauren M. Cohee ${ }^{1,8 凶}$, Clarissa Valim 2,8, Jenna E. Coalson ${ }^{3,7}$, Andrew Nyambalo ${ }^{4}$, \\ Moses Chilombe ${ }^{5}$, Andrew Ngwira ${ }^{5}$, Andy Bauleni ${ }^{5}$, Karl B. Seydel ${ }^{4,6}$, Mark L. Wilson ${ }^{3}$, \\ Terrie E. Taylor ${ }^{4,6}$, Don P. Mathanga ${ }^{5}$ \& Miriam K. Laufer ${ }^{1}$
}

In areas where malaria remains entrenched, novel transmission-reducing interventions are essential for malaria elimination. We report the impact screening-and-treatment of asymptomatic Malawian schoolchildren ( $n=364$ in the rainy season and 341 in the dry season) had on gametocyte-the parasite stage responsible for human-to-mosquito transmission-carriage. We used concomitant householdbased surveys to predict the potential reduction in transmission in the surrounding community. Among 253 students with $P$. falciparum infections at screening, 179 (71\%) had infections containing gametocytes detected by Pfs 25 qRT-PCR. 84\% of gametocyte-containing infections were detected by malaria rapid diagnostic test. While the gametocyte prevalence remained constant in untreated children, treatment with artemether-lumefantrine reduced the gametocyte prevalence $(p<0.0001)$ from 51.8 to $9.7 \%$ and geometric mean gametocyte density $(p=0.008)$ from 0.52 to 0.05 gametocytes/ microliter. In community surveys, $46 \%$ of all gametocyte-containing infections were in school-age children, who comprised only $35 \%$ of the population. Based on these estimates six weeks after the intervention, the gametocyte burden in the community could be reduced by $25-55 \%$ depending on the season and the measure used to characterize gametocyte carriage. Thus, school-based interventions to treat asymptomatic infections may be a high-yield approach to not only improve the health of schoolchildren, but also decrease malaria transmission.

In the last two decades, many regions have made substantial progress toward elimination of malaria. Recently, however, progress has stalled and malaria remains entrenched in some highly endemic areas like Malawi ${ }^{1}$. In these areas, standard interventions (e.g., vector control, universal access to effective testing and treatment, and preventive treatment of high risk population ${ }^{2}$ ) may not adequately interrupt human-to-mosquito Plasmodium falciparum transmission, which is required to move from malaria control toward malaria elimination ${ }^{3}$. Asymptomatic infections in individuals at lower risk of disease often remain untreated, creating a silent reservoir that perpetuates transmission ${ }^{4,5}$. To achieve malaria elimination, interventions must not only prevent and/or treat disease, they must also decrease parasite transmission ${ }^{6}$.

Primary school-age children (6-to-15 year olds) bear an under-appreciated burden of $P$. falciparum infection and they may be an important human infectious reservoir for malaria ${ }^{7,8}$. School-age children frequently have higher prevalence of infection than younger children and adults ${ }^{9-13}$ and their infections more often contain gametocytes, the parasite stage required for human-to-mosquito transmission ${ }^{11,14}$. When bitten, infected school-age children and young children are similarly infectious to mosquitoes ${ }^{15}$. However, school-age children have larger body surface area and less bed net use compared to younger children, increasing the odds of being bitten by mosquitos $^{16-19}$. Together these factors suggest school-age children are significant sources of human-to-mosquito P. falciparum transmission ${ }^{8,19,20}$. Indeed, expanding community-based seasonal malaria chemoprevention from

${ }^{1}$ Center for Vaccine Development and Global Health, University of Maryland School of Medicine, Baltimore, MD 21201, USA. 2Department of Global Health, Boston University School of Public Health, Boston, MA, USA. ${ }^{3}$ Department of Epidemiology, School of Public Health, University of Michigan, Ann Arbor, MI, USA. ${ }^{4}$ Blantyre Malaria Project, University of Malawi College of Medicine, Blantyre, Malawi. ${ }^{5}$ Malaria Alert Center, University of Malawi College of Medicine, Blantyre, Malawi. ${ }^{6}$ College of Osteopathic Medicine, Michigan State University, East Lansing, MI, USA. ${ }^{7}$ Present address: Eck Institute for Global Health, University of Notre Dame, Notre Dame, IN, USA. ${ }^{8}$ These authors contributed equally: Lauren M. Cohee and Clarissa Valim. ${ }^{\square}$ email: Icohee@ som.umaryland.edu 


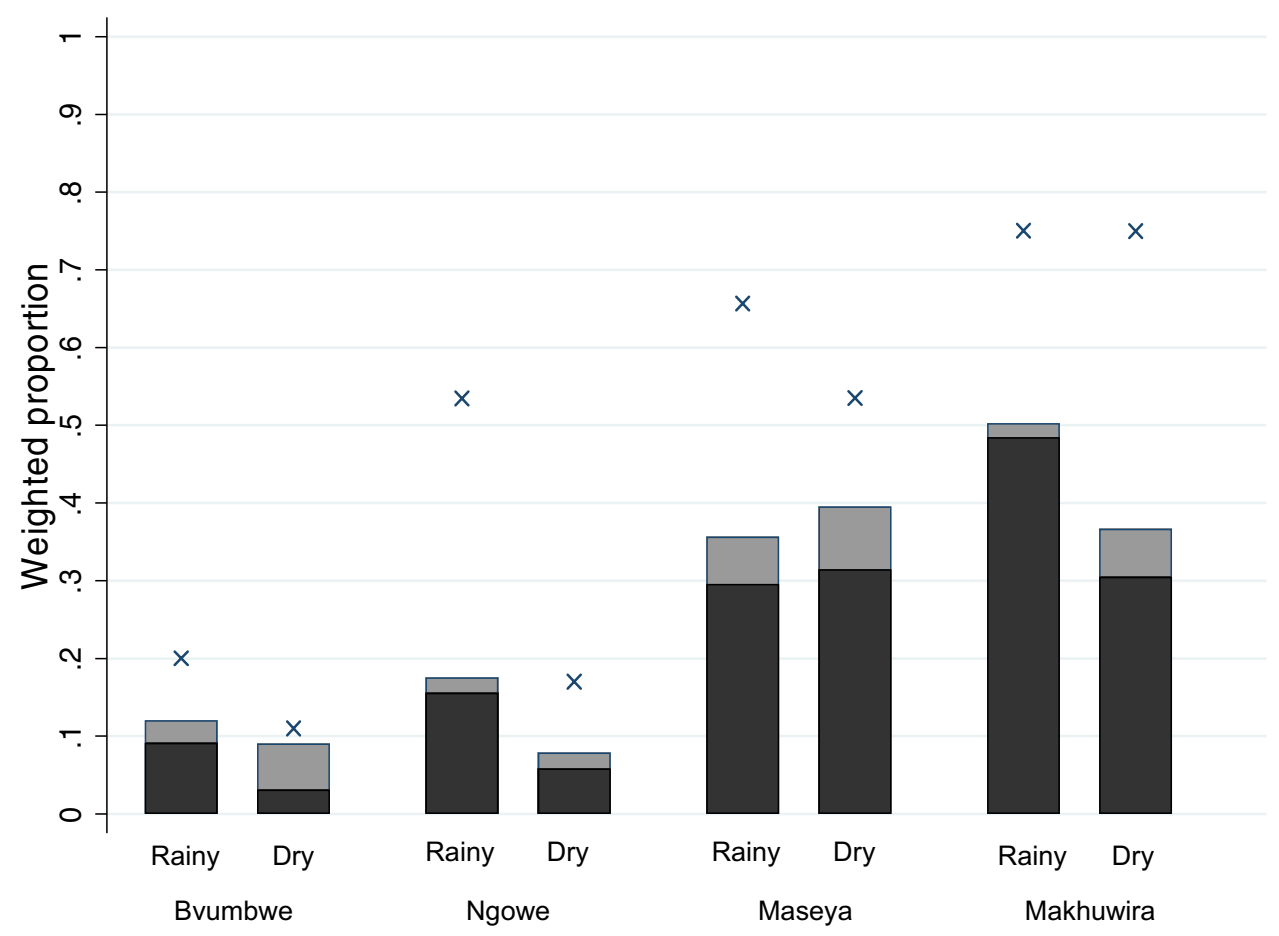

Figure 1. Baseline weighted proportion of students with $P$. falciparum infection detected by PCR $(\times)$ and the weighted proportion of students with gametocyte-containing infections detected (black bar) and not detected (grey bar) by rapid diagnostic test in school-based cohorts.

only young children to include school-age children was associated with a $20 \%$ decrease in clinical malaria in older community members who did not receive the intervention ${ }^{21}$.

With rising school enrollments across the malaria-endemic world, existing school infrastructure, such as multi-sectoral programs for school feeding and deworming programs, could be leveraged to deliver malaria interventions to school-age children. Approaches to deliver preventive treatment include "intermittent preventive treatment" where all students regularly receive antimalarial drugs for parasite clearance and transient prophylaxis, and "screening-and-treatment" where all students are screened and only those testing positive receive treatment. Preventive treatment directly decreases infection and gametocyte prevalence, clinical disease, and anemia among students $^{22,23}$, but potential indirect effects on the surrounding community have not been fully assessed. While dynamic modeling data suggests the utility of this intervention, only one study has reported the impact of schoolbased preventive treatment on community-level infection, finding a small but significant decrease in community infection prevalence surrounding intervention schools despite low intervention coverage ${ }^{24,25}$.

To evaluate the potential impact of school-based malaria interventions on community transmission, we conducted a school-based screen-and-treat cohort study in the southern region of Malawi concurrent with household-based cross-sectional surveys. Primary school students in four schools were screened for infection using a rapid diagnostic test (RDT). If positive, students were treated with artemether-lumefantrine. Subsequent gametocyte carriage in treated and untreated students was assessed by qRT-PCR after one, two, and six weeks. Using the age distribution of gametocyte carriers in the communities surrounding the school, we estimated the potential reduction in prevalence of gametocyte-containing infections and gametocyte density in the community following screening-and-treatment of schoolchildren. If school-based preventive treatment substantially reduces gametocyte prevalence and density in the community, this intervention could both improve the health of schoolchildren and contribute more broadly to malaria elimination.

\section{Results}

We enrolled 786 students in school-based cohorts: 405 in the rainy season and 381 in the dry season. Complete follow-up data were obtained for 616 students (78\% of enrolled students) (Supplemental Figure S2). Among the 705 students who attended the baseline visits and underwent screening $(n=364$ in the rainy season and 341 in the dry season), the mean age was 10.4 years, $51 \%$ were female, and $47 \%$ reported sleeping under a net the previous night. The weighted prevalence of RDT-positivity among students at baseline was $42 \%$. An additional $8 \%$ of students had infection detected by only PCR (all parasite stages). The distribution of infection prevalence varied by school and season (Fig. 1).

Determinants of gametocyte burden in school-based cohorts at baseline. Overall, at baseline, 28\% (unweighted 179/701, 4 missing baseline gametocyte results) of students had gametocyte-containing infec- 


\begin{tabular}{|c|c|c|c|c|c|c|c|}
\hline \multirow[b]{2}{*}{ Predictor } & \multirow[b]{2}{*}{ N PCR positive } & \multirow[b]{2}{*}{$\begin{array}{l}\mathrm{N} \text { gametocyte } \\
\text { positive }\end{array}$} & \multirow{2}{*}{$\begin{array}{l}\text { Weighted } \dagger \\
\text { proportion } \\
\text { containing } \\
\text { gametocytes }\end{array}$} & \multicolumn{2}{|c|}{ Bivariate association $^{*}$} & \multicolumn{2}{|c|}{ Multivariable association ${ }^{*}$} \\
\hline & & & & OR $[95 \% \mathrm{CI}]$ & p-value & OR $[95 \% \mathrm{CI}]$ & p-value \\
\hline \multicolumn{8}{|l|}{ Site/school ${ }^{\mathrm{a}}$} \\
\hline Bvumbwe & 21 & 19 & 0.89 & Ref & 0.19 & Ref & 0.40 \\
\hline Ngowe & 35 & 24 & 0.72 & $0.30[0.05-1.8]$ & & $0.35[0.05-2.4]$ & \\
\hline Maseya & 93 & 71 & 0.76 & $0.37[0.07-2.0]$ & & $0.44[0.07-2.7]$ & \\
\hline Makhuwira & 104 & 65 & 0.64 & $0.21[0.04-1.1]$ & & $0.28[0.05-1.6]$ & \\
\hline \multicolumn{8}{|l|}{ Season } \\
\hline Dry & 123 & 77 & 0.63 & Ref & 0.07 & Ref & 0.040 \\
\hline Rainy & 130 & 102 & 0.77 & $1.9[0.95-3.6]$ & & $2.0[1.0-4.0]$ & \\
\hline Age (years) & 253 & 179 & $-{ }^{\mathrm{b}}$ & $0.90[0.79-1.0]$ & 0.10 & $0.89[0.78-1.0]$ & 0.08 \\
\hline \multicolumn{8}{|l|}{ Sex } \\
\hline Male & 129 & 92 & 0.71 & Ref & 0.70 & & \\
\hline Female & 124 & 87 & 0.69 & $0.88[0.46-1.7]$ & & & \\
\hline \multicolumn{8}{|l|}{ Hemoglobin } \\
\hline Not anemic & 197 & 137 & 0.67 & Ref & 0.16 & & \\
\hline Anemic $^{\mathrm{c}}$ & 55 & 41 & 0.77 & $1.8[0.79-4.1]$ & & & \\
\hline \multicolumn{8}{|c|}{ Fever status $^{\mathrm{d}}$} \\
\hline Afebrile & 109 & 78 & 0.72 & Ref & 0.88 & & \\
\hline Febrile & 144 & 101 & 0.68 & $0.95[0.48-1.9]$ & & & \\
\hline \multicolumn{8}{|c|}{ Recent treatment $^{\mathrm{e}}$} \\
\hline Not treated & 238 & 168 & 0.70 & Ref & 0.99 & & \\
\hline Treated & 13 & 10 & 0.70 & $0.99[0.20-4.9]$ & & & \\
\hline \multicolumn{8}{|c|}{ Slept under a net ${ }^{\mathrm{f}}$} \\
\hline No & 151 & 102 & 0.68 & Ref & 0.58 & & \\
\hline Yes & 97 & 73 & 0.72 & $1.2[0.61-2.4]$ & & & \\
\hline
\end{tabular}

Table 1. Baseline prevalence and predictors of infections containing gametocytes among students with $P$. falciparum infections in the school-based cohort. ${ }^{\dagger}$ Survey weights were calculated for each stratum, defined as each grade within each school (details in Supplemental Information). ${ }^{*}$ Obtained in a survey weighted logistic regression that analyzed the variables shown in the table. $\mathrm{OR}=$ Odds Ratio; $\mathrm{CI}=$ Confidence Interval. Bold values are statistically significant for $\mathrm{p}$-values $<0.05$. ${ }^{a}$ Univariate association for school, all other associations include school. Prevalence setting: Bvumbwe-low, seasonal; Ngowe-moderate, seasonal; Maseya-high; Makhuwira-high. ${ }^{b}$ Mean age of students with infections containing gametocytes was 10.9 years compared to 11.6 years among those whose infections did not contain gametocytes. ${ }^{c}$ Hemoglobin $<11.0 \mathrm{~g} / \mathrm{dL} ; 1 \mathrm{missing}$ observation. ${ }^{\mathrm{d}}$ Measured temperature $\geq 37.5^{\circ} \mathrm{C}$ at visit or reported in the last two weeks. ${ }^{\mathrm{e}}$ Reported in the last two weeks; 2 missing observations. ${ }^{\mathrm{f}} 5$ missing observations.

tions (8-50\% across schools and seasons; Fig. 1). To disentangle predictors of having an infection containing gametocytes from those of simply having any $P$. falciparum infection, we evaluated predictors among only students with $P$. falciparum infection. Among the 253 students with $P$. falciparum infection by PCR at baseline, $70 \%$ contained gametocytes detected by Pfs 25 qRT-PCR. Infections were more likely to contain gametocytes in the rainy season and in younger students, although the age association was not statistically significant (Table 1). Associations were comparable in multivariable models with or without non-significant variables.

The crude weighted geometric mean gametocyte density among those with gametocytes was 0.34 gametocytes/ $\mu \mathrm{l}$ (95\% CI: 0.19-0.60 gametocytes/ $\mu \mathrm{l}$; range 0.002-661 gametocytes/ $\mu$ l). Gametocyte density was higher in the rainy season and decreased with age (Table 2). Among all students at baseline, 5.3\% (unweighted 32/701) had infections with $\geq 10$ gametocytes/ $\mu$, which are associated with increased likelihood of transmission ${ }^{26}$. Among students with gametocyte-containing infections, $19 \%$ (unweighted 32/179) of infections contained $\geq 10$ gametocytes/ $\mu$ l. In addition to season and age, odds of having $\geq 10$ gametocytes/ $\mu$ l were significantly higher in children with fever and in the lowest prevalence school (Bvumbwe; Supplemental Table S3).

Gametocyte-containing infections missed by RDT screening. A key factor in the success of screenand-treat interventions is the screening test sensitivity for detecting infections of interest, i.e., gametocyte-containing infections for transmission reduction. Sixteen percent (unweighted 38/180) of all students with gametocyte-containing infections and $9 \%$ (unweighted 4/32) with infections containing $\geq 10$ gametocytes/ $\mu$ l were RDT-negative and thus not treated. RDTs were less likely to detect gametocyte-containing infections at lower densities (Table 3). After adjusting for density, RDTs also missed gametocyte-containing infections more often in the lowest prevalence school (Bvumbwe) than other schools (OR for failure 6.6, 95\% CI: 1.9-24, p=0.004). 


\begin{tabular}{|c|c|c|c|c|c|}
\hline \multirow[b]{2}{*}{ Predictor } & \multirow[b]{2}{*}{ N gametocyte positive } & \multicolumn{2}{|l|}{ Bivariate associations $^{*}$} & \multicolumn{2}{|c|}{ Multivariable associations ${ }^{*}$} \\
\hline & & \% Difference ${ }^{* *}[95 \% \mathrm{CI}]$ & p-value & \% Difference $[95 \% \mathrm{CI}]$ & p-value \\
\hline \multicolumn{6}{|l|}{ Site/school ${ }^{\mathrm{a}}$} \\
\hline Bvumbwe & 19 & Ref & 0.36 & Ref & 0.20 \\
\hline Ngowe & 24 & $-39[-93$ to 470$]$ & & $-50[-89$ to 123$]$ & \\
\hline Maseya & 71 & $-63[-95$ to 170$]$ & & $-37[-81$ to 108$]$ & \\
\hline Makhuwira & 65 & $-79[-97$ to 70$]$ & & $-70[-91$ to 1$]$ & \\
\hline \multicolumn{6}{|l|}{ Season } \\
\hline Dry & 77 & Ref & $<0.001$ & Ref & $<0.001$ \\
\hline Rainy & 102 & $4740[2210$ to 10040$]$ & & $5186[2447$ to 10870$]$ & \\
\hline Age (per year increase) & 179 & $-12[-29$ to 10$]$ & 0.25 & $-18[-30$ to -3$]$ & 0.02 \\
\hline \multicolumn{6}{|l|}{ Sex } \\
\hline Male & 92 & Ref & 0.12 & & \\
\hline Female & 87 & $150[-20$ to 710$]$ & & & \\
\hline \multicolumn{6}{|l|}{ Hemoglobin } \\
\hline Not anemic & 137 & Ref & 0.42 & & \\
\hline Anemic $^{b}$ & 41 & $80[-58$ to 670$]$ & & & \\
\hline \multicolumn{6}{|l|}{ Fever status $^{\mathrm{c}}$} \\
\hline Afebrile & 78 & Ref & 0.13 & & \\
\hline Febrile & 101 & $120[-20$ to 510$]$ & & & \\
\hline \multicolumn{6}{|l|}{ Recent treatment $^{\mathrm{d}}$} \\
\hline Not treated & 168 & Ref & 0.21 & & \\
\hline Treated & 10 & $340[-56$ to 4240$]$ & & & \\
\hline \multicolumn{6}{|l|}{ Slept under a net ${ }^{\mathrm{e}}$} \\
\hline No & 102 & Ref & 0.30 & & \\
\hline Yes & 73 & $90[-43$ to 510$]$ & & & \\
\hline
\end{tabular}

Table 2. Predictors of baseline gametocyte density among infections containing gametocytes in the school cohort. ${ }^{\star}$ Obtained in a survey weighted (weights calculated by school ${ }^{\star}$ standard) linear regression with logarithm transformed gametocyte density as an outcome, and analyzing the variables shown in the table. ${ }^{*}$ Percent difference in gametocyte density from reference category is calculated as: [exp $\left.(\operatorname{coeff})-1\right] \times 100$. $\mathrm{OR}=$ Odds Ratio; $\mathrm{CI}=$ Confidence Interval. Bold values are statistically significant for $\mathrm{p}$-values $<0.05$. ${ }^{a}$ Univariate association for school, all bivariate include adjustment for school. ${ }^{\mathrm{b}}$ Hemoglobin $<11.0 \mathrm{~g} / \mathrm{dL} ; 1$ missing observation. ${ }^{\mathrm{c}}$ Measured temperature $\geq 37.5^{\circ} \mathrm{C}$ at baseline visit or reported in the last two weeks.

${ }^{\mathrm{d}}$ Reported in the last two weeks; 1 missing observations. ${ }^{\mathrm{e}} 4$ missing observations.

Impact of screen-and-treat intervention on gametocyte prevalence and density in the school-based cohort. As hypothesized, treatment decreased gametocyte prevalence and density among students during follow-up. Comparing gametocyte prevalence among all students from baseline to six weeks after the intervention there was a reduction from $31.1 \%$ (95\% CI: $27.6,34.5)$ to $12.8 \%(95 \%: 9.5,16.1)$ in the rainy season and from 25.7 (95\% CI: 20.9, 30.4) to 8.8\% (95\%: 5.4, 12.2) in the dry season. Geometric mean gametocyte density decreased from 0.50 gametocytes/microliter (95\% CI: $0.09,2.81)$ to $0.02(95 \%: 0.002,0.20)$ in the rainy season and 0.003 (95\% CI: $0.001,0.009)$ to 0.0001 (95\%: $0.00001,0.002)$ in the dry season.

The overall reduction occurred among treated (RDT positive) students, while prevalence and density among untreated (RDT negative) students remained constant or increased. The prevalence of gametocyte-containing infections among treated students decreased from 52\% (95\% CI: 45-58\%) at baseline to 11\% (95\% CI: 7-14\%) after two weeks, a $79 \%$ reduction (Fig. 2a). Gametocyte prevalence remained low after six weeks $[10 \%, 95 \%$ CI: 6-14\%)]. Among untreated students (RDT-negative), the prevalence of gametocyte-containing infections remained unchanged from baseline [9\% (95\% CI: 5-14\%)] to six weeks after the intervention [12\% (95\% CI: 7-17\%)].

Gametocyte density followed the same trend among the treated students, with $90 \%$ reduction in the geometric mean of gametocyte density after treatment, but increased by $59 \%$ in untreated children (Fig. $2 \mathrm{~b}$ ). There were 32 students with infections containing $\geq 10$ gametocytes/ $\mu$ at baseline: 28 in RDT-positive students and four in RDT-negative students. Two weeks after screening-and-treatment, only one infection containing $\geq 10$ gametocytes/ $\mu$ l was detected, which was in an untreated student. Six weeks after treatment there were four infections containing $\geq 10$ gametocytes/ $\mu \mathrm{l}$, two in students that were not treated and two in students that received treatment, corresponding to a $90 \%$ reduction in prevalence. Results were similar across schools, a proxy for transmission settings (Fig. 2c-f).

Gametocytemia in the school-based cohort compared to school-age children in the community. The proportion of infections containing gametocytes in the school-based cohort was similar to that in the 862 school-age children tested in the community $(25 \%$ vs. $27 \%, p=0.62)$, supporting the generalizability of 


\begin{tabular}{|c|c|c|c|c|c|c|c|}
\hline \multirow[b]{2}{*}{ Predictor } & \multirow[b]{2}{*}{ N gametocyte positive } & \multirow[b]{2}{*}{ N RDT negative } & \multirow{2}{*}{$\begin{array}{l}\text { Weighted } \dagger \text { proportion not detected } \\
\text { by RDT }\end{array}$} & \multicolumn{2}{|c|}{ Bivariate association $^{*}$} & \multicolumn{2}{|c|}{ Multivariable association $^{*}$} \\
\hline & & & & OR $[95 \% \mathrm{CI}]$ & p-value & OR $[95 \% \mathrm{CI}]$ & p-value \\
\hline \multicolumn{8}{|l|}{ Site/school ${ }^{\mathrm{a}}$} \\
\hline Bvumbwe & 19 & 8 & 0.40 & Ref & 0.059 & Ref. ${ }^{g}$ & 0.01 \\
\hline Ngowe & 25 & 4 & 0.15 & $0.27[0.05-1.4]$ & & $0.21[0.04-1.2]$ & \\
\hline Maseya & 71 & 15 & 0.19 & $0.34[0.10-1.2]$ & & $0.25[0.06-0.98]$ & \\
\hline Makhuwira & 65 & 10 & 0.10 & $0.16[0.04-0.60]$ & & $0.09[0.02-0.38]$ & \\
\hline \multicolumn{8}{|l|}{ Season } \\
\hline Rainy & 103 & 15 & 0.10 & Ref & & & \\
\hline Dry & 77 & 22 & 0.23 & $2.9[1.2-7.0]$ & 0.02 & & \\
\hline Age (per year increase) & 180 & 37 & $-{ }^{\mathrm{b}}$ & $1.1[0.96-1.3]$ & 0.13 & & \\
\hline Gametocyte density $(\log 10)$ & 180 & 37 & $-^{c}$ & $0.76[0.64-0.90]$ & 0.002 & $0.76[0.64-0.90]$ & 0.002 \\
\hline \multicolumn{8}{|l|}{ Sex } \\
\hline Male & 92 & 16 & 0.13 & Ref & 0.25 & & \\
\hline Female & 88 & 21 & 0.20 & $1.6[0.71-3.7]$ & & & \\
\hline \multicolumn{8}{|l|}{ Hemoglobin } \\
\hline Not anemic & 138 & 31 & 0.17 & Ref & 0.85 & & \\
\hline Anemic $^{\mathrm{d}}$ & 41 & 6 & 0.13 & $0.90[0.30-2.7]$ & & & \\
\hline \multicolumn{8}{|l|}{ Fever status $^{e}$} \\
\hline Afebrile & 78 & 20 & 0.21 & Ref & 0.36 & & \\
\hline Febrile & 102 & 17 & 0.12 & $0.68[0.30-1.6]$ & & & \\
\hline \multicolumn{8}{|l|}{ Recent treatment $^{\mathrm{f}}$} \\
\hline Not treated & 169 & 36 & 0.17 & Ref & 0.41 & & \\
\hline Treated & 10 & 1 & 0.07 & $0.41[0.05-3.4]$ & & & \\
\hline \multicolumn{8}{|l|}{ Slept under a net ${ }^{\mathrm{h}}$} \\
\hline No & 102 & 17 & 0.13 & Ref & 0.42 & & \\
\hline Yes & 74 & 18 & 0.17 & $1.4[0.59-3.5]$ & & & \\
\hline
\end{tabular}

Table 3. Characteristics of gametocyte containing infections missed (not detected) by RDT screening at baseline. ${ }^{\dagger}$ Survey weights were calculated for each stratum, defined as each grade within each school (details in Supplemental Information). ${ }^{\star}$ Obtained in a survey weighted logistic regression of gametocyte-containing infections with the outcome of RDT negativity. All analyzed variables are shown in the table. OR=Odds Ratio; $\mathrm{CI}=$ Confidence Interval. Bold values are statistically significant for $\mathrm{p}$-values $<0.05$. ${ }^{\mathrm{a}}$ Univariate association for school, all other associations include school. Prevalence setting: Bvumbwe-low, seasonal; Ngowe-moderate, seasonal; Maseya-high; Makhuwira-high. ${ }^{\mathrm{b}}$ Mean age of students with gametocyte containing infections not detected by RDT was 11.5 compared to 10.8 years for students with gametocyte containing infections detected by RDT. ${ }^{c}$ Among students with gametocyte-containing infections not detected by RDT, the mean $\log$ gametocyte density was -2.417 compared to a mean log gametocyte density of -0.825 among students with gametocyte-containing infections detected by RDT. ${ }^{\mathrm{d}}$ Hemoglobin $<11.0 \mathrm{~g} / \mathrm{dL} ; 1 \mathrm{missing}$ observation. ${ }^{\mathrm{e}}$ Measured temperature $\geq 37.5^{\circ} \mathrm{C}$ at baseline visit or reported in the last two weeks. ${ }^{\mathrm{f}}$ Reported in the last two weeks; 1 missing observation. ${ }^{\mathrm{g}}$ Adjusted OR for Bvumbwe compared to all other schools in the multivariable model is 6.6 [95\% CI: $1.9-24], \mathrm{p}=0.004{ }^{\mathrm{h}} 4$ missing observations.

the intervention results to all school-age children in the community. The geometric mean density was higher in school-age children in the community (0.99 gametocytes/ $\mu$ l [95\% CI: $0.71-1.38])$ than in the school $(0.34$ [95\% CI: $0.19-0.60], p=0.03)$. However, the proportion of infections containing $\geq 10$ gametocytes/ $\mu$ l among those with gametocytes was similar ( $20 \%$ in the community vs. $18 \%$ in schools, $\mathrm{p}=0.51$ ).

Predicted reduction in the community gametocyte population following treatment in schools. The screen-and-treat intervention reduced gametocytemia in our school-based cohorts. Furthermore, school-age children contributed substantially to the population of gametocyte-carriers in the surrounding communities; $46 \%$ of all gametocyte-containing infections (229/494) and infections containing $\geq 10$ gametocytes/ $\mu \mathrm{l}(47 / 103)$ were in school-age children, who comprised only $35 \%$ of the population (Supplemental Text S4 and Figure S3). If the screen-and-treat intervention were extended to all school attendees, we estimate it could result in at least six weeks of reductions in the community prevalence of gametocyte-containing infections as large as $26 \%$ in the rainy and $34 \%$ in the dry season (Fig. 3a and b). The total gametocyte burden (sum of gametocyte densities) in the community would be reduced by $33 \%$ in the rainy season and $25 \%$ in the dry season (Fig. $3 \mathrm{c}$ and $\mathrm{d}$ ). The number of infections containing $\geq 10$ gametocytes/ $\mu$ l would be reduced by $44 \%$ and $55 \%$ in the rainy and dry seasons, respectively (Fig. 3e and f). Reductions occurred in both high and low transmission settings, though the reductions were larger in the high transmission settings (Supplemental Information Figure S4). 
a

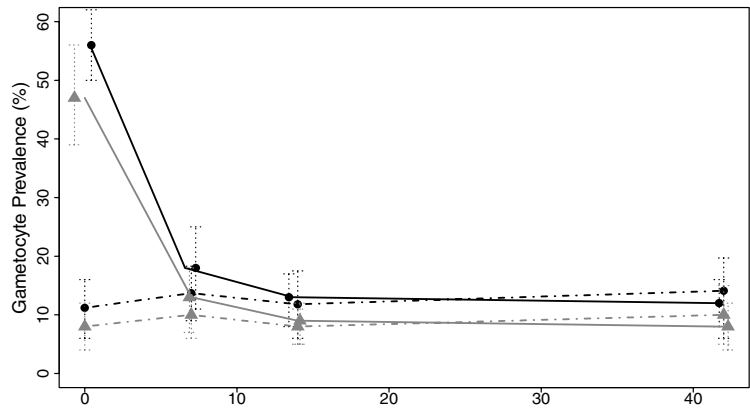

C

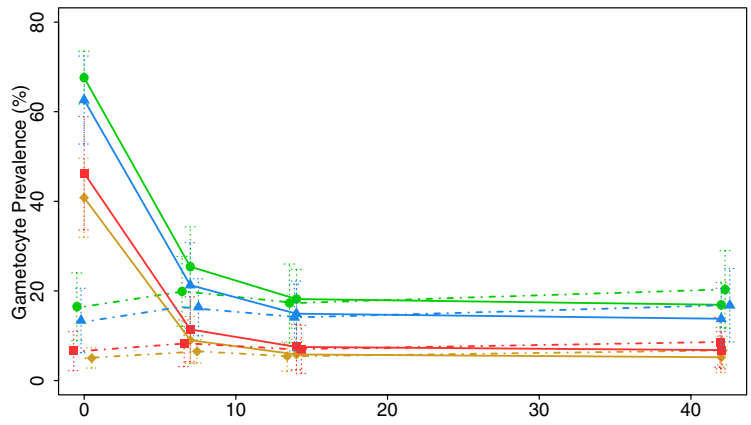

e

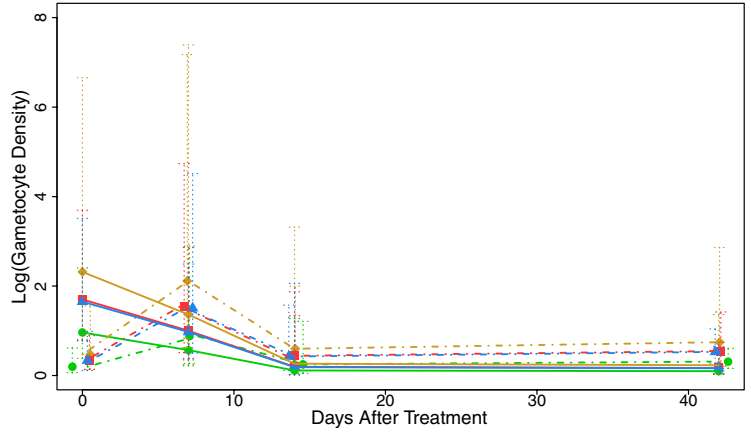

b

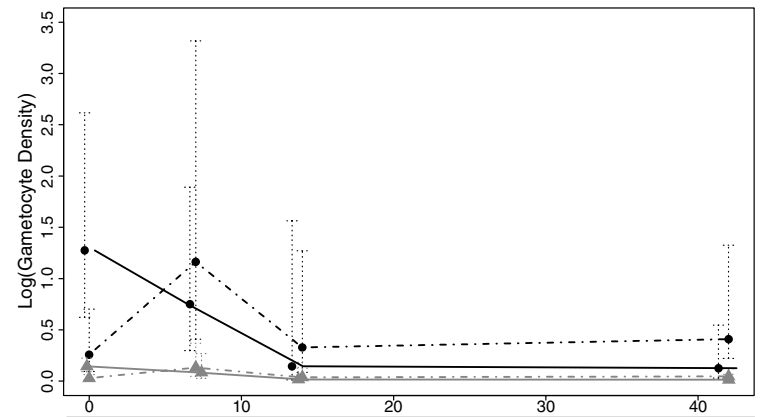

d

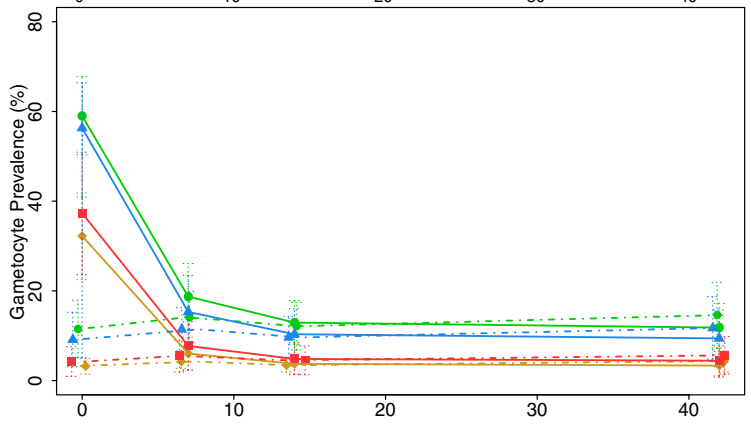

$f$

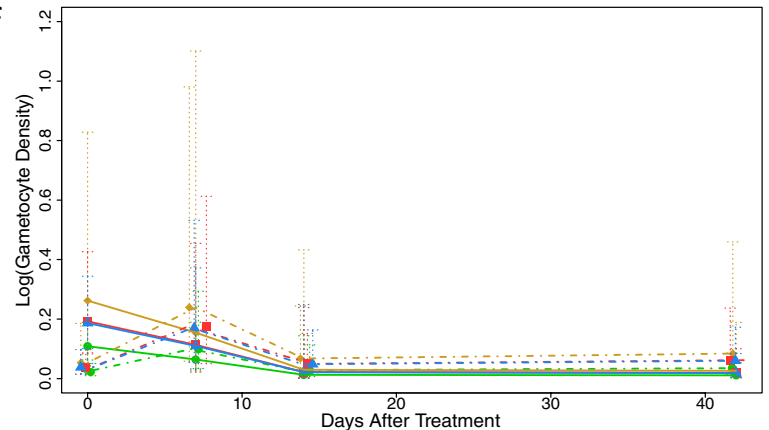

Figure 2. Impact of treating RDT positive students on the proportion and density of gametocytes in the schoolbased cohort over time. Proportion of students with gametocytes in all schools combined (a) and by school in the rainy $(\mathrm{c})$ and the dry $(\mathrm{d})$ season. Mean gametocyte density (logarithm transformed) among students in all schools (b) and by school in the rainy (e) and the dry (f) season. Solid lines represent students who received treatment; Dashed lines represent students who did not receive treatment. Color designates season in $\mathrm{a}$ and $\mathrm{b}$ rainy (black) and dry (gray). Color designates school in c-f Bvumbwe (yellow), Ngowe (red), Maseya (blue), Makhuwira (green). Both estimates were obtained in random effects longitudinal analysis (full models in Supplemental Tables S3 and S4).

\section{Discussion}

In countries like Malawi where current malaria control measures have not yielded substantial reduction in $P$. falciparum transmission, in addition to optimizing current measures, new approaches are needed to target transmission ${ }^{3}$. Our results support the use of school-based screening-and-treatment as an additional intervention that may not only improve the health of schoolchildren, but also decrease malaria transmission in the community. Almost half of high-density gametocyte-containing infections in the community were in school-age children; school-based treatment nearly eliminated this group's contribution to transmission for up to six weeks. We also showed that RDTs detected most gametocyte-containing infections that are likely to be transmitted, and that treatment of asymptomatic infections based on RDT results reduced gametocyte prevalence and density. Treating only children with positive RDTs could reduce the community-level gametocyte burden by a third. This reduction in potentially infectious reservoirs could help interrupt persistent malaria transmission.

The high prevalence of gametocyte-containing infections in school-age children in this study is consistent with prior work by our group and others ${ }^{11,14,19}$. However, to our knowledge, this is the first study to simultaneously determine gametocyte burden in schools and surrounding communities to estimate the potential impact of school-based treatment on transmission. In Uganda, school-based intermittent preventive treatment was associated with reduced parasite prevalence in the surrounding community in a large cluster randomized trial ${ }^{25}$. Although the community-level effect was statistically significant, the magnitude was limited by low intervention coverage. Because the study in Uganda was conducted as a clinical trial, parents had to come to the school to consent for each student, creating logistical barriers. Thus, further estimates of the full potential of this intervention are needed. 
a.

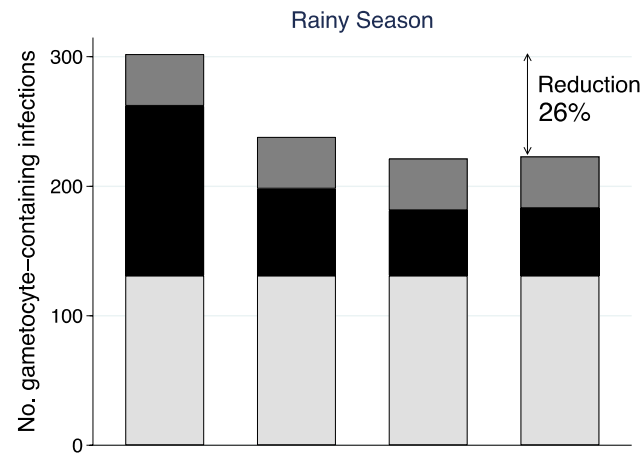

C.

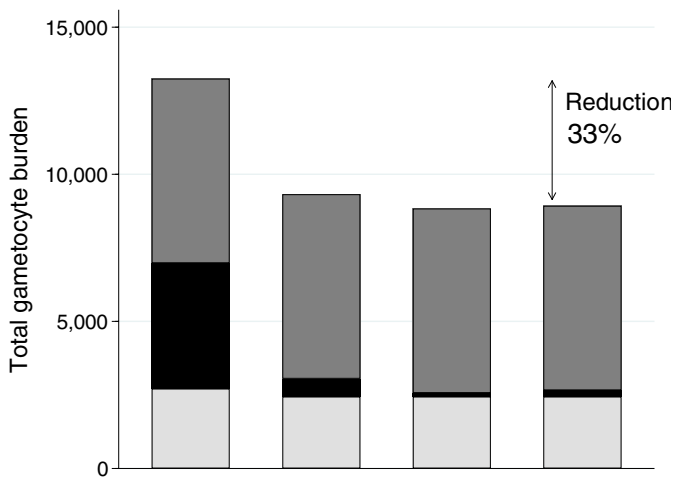

e.

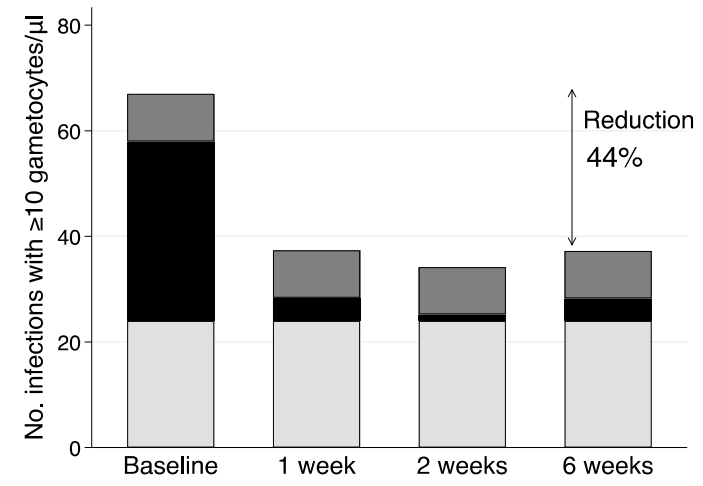

b. 300

Dry Season

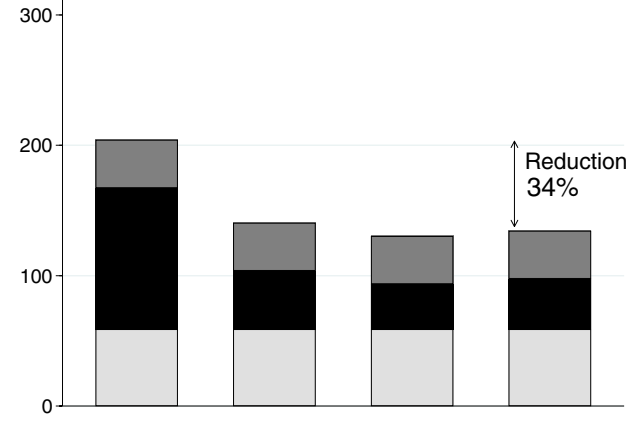

d. 3,000

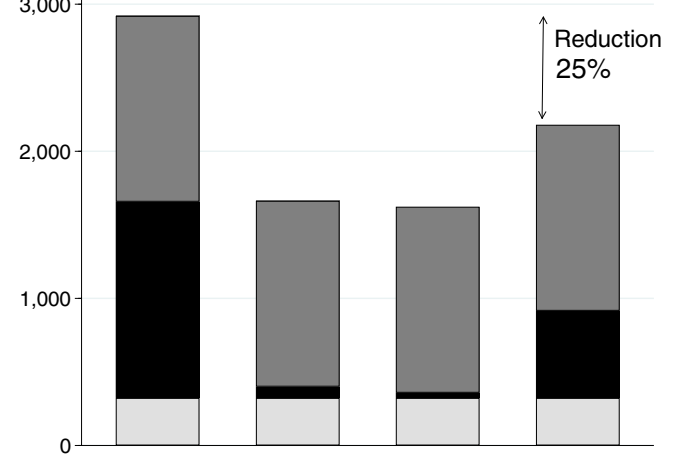

f.

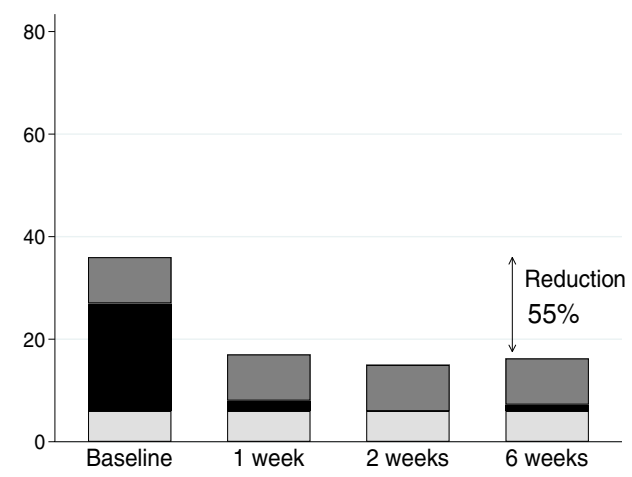

Figure 3. Predicted impact of school-based treatment of RDT positive students on the population of gametocytes in the surrounding community. Following school-based screening-and-treatment at baseline, the estimated impact on gametocyte prevalence ( $\mathrm{a}$ in rainy and $\mathrm{b}$ in dry seasons), total gametocyte burden (c in rainy and $\mathrm{d}$ in dry seasons), and number of infections containing $\geq 10$ gametocytes/ $\mu \mathrm{l}$ ( $\mathrm{e}$ in rainy and $\mathrm{f}$ in dry seasons) in the communities surrounding the schools are predicted at one, two, and six weeks after the intervention. Color indicates the proportion of the gametocyte measure by age group: school-age children (6-15y) - black; younger children (6 m-5y) — light grey; adults ( $>15 y)$-dark grey. Total gametocyte burden is the sum of gametocyte densities in individuals in each age group. These calculations assume treatment is not provided to young children, adults, or school-age children who test negative by RDT when the intervention is implemented. Reduction is calculated as the proportional difference between the baseline and six-weeks post intervention.

While our results lend further support the potential contribution of school-based preventive treatment to transmission reduction, there are limitations to our analyses. We did not use RDTs to detect infections in the communities surrounding schools and had to extrapolate from the relationships of PCR- and RDT-detected infections in the school-based cohort to estimate the number of RDT-positive children who would attend school from the community. However, the distribution of gametocyte prevalence in the school-based and communitybased surveys was comparable, supporting the estimate. Furthermore, we used sensitive molecular methods to detect and quantify gametocytes but did not directly measure infectiousness using feeding assays. However, our data still likely represent the relative contribution of different age groups to transmission because we evaluated not only the prevalence of gametocytes but the density and further evaluate high-density gametocyte containing infections, which are the most likely to lead to mosquito infection ${ }^{26}$. Furthermore, prior studies have shown that school-age children and younger children are more infectious to mosquitos than adults ${ }^{15}$ and school-age children 
are more likely than younger children to be bitten by competent vectors ${ }^{19}$. Lastly, our estimates of the impact of the intervention in the community assume universal coverage in students. In Malawi, school-based deworming programs routinely report coverage of $>90 \%$ of school-age children ${ }^{27}$. Thus high levels of coverage are possible.

Our results likely underestimate the overall effect of school-based treatment for three reasons. First, the impact of clearing gametocyte-containing infections in school-age children is expected to be amplified because competent vectors probably feed more often on school-age children and adults who have larger body surface than younger children ${ }^{18,28}$ and use bed nets less frequently ${ }^{17-19,28}$. Second, in our community-level predictions, we conservatively assumed that gametocyte-containing infections in non-treated age groups would remain constant. However, the decreased pool of infectious school-age children should also lead to fewer new infections and fewer gametocyte-containing infections in younger children and adults. Third, our results are based on a single treatment intervention; the impact of repeated school-based treatment is likely to be even larger. In clinical trials evaluating the impact of school-based malaria preventive treatment on health outcomes, the most successful interventions were repeated either monthly during the high transmission season or quarterly throughout the year ${ }^{22}$. Reinfection rates, seasonal variation in transmission, and duration of the prophylactic effect of the treatment drugs should be used to guide the frequency of intervention required.

Currently, schools are platforms for successful deworming campaigns and feeding programs, and a growing number of interventions target this age group, including vitamin supplementation and adolescent vaccinations. The vast majority of children in sub-Saharan Africa attend school, making schools a logistically feasible platform for targeted interventions. In our setting, 91\% (range 82-99\% depending on community and season) of school-age children in the community attended school. Schools are particularly important to reaching this age group, since children rarely have routine contact with the health care system after completing childhood immunizations. Adding malaria preventive treatment to other school-based interventions in an integrated school health package should improve the cost:benefit ratio, promote sustainability, and improve health care access to this underserved population.

One concern is that risk of infection and disease could increase once students leave school or if the intervention ceased. A prior study of chemoprophylaxis in younger children demonstrated a transient increase in clinical infection when the intervention was discontinued ${ }^{29}$. However, this "rebound effect" was not observed in all chemoprophylaxis trials, nor has it been found after intermittent preventive treatment of infants ${ }^{30-32}$. In highly endemic settings where school-based treatment interventions are most needed, schoolchildren have acquired partial immunity and often have sub-clinical or asymptomatic infections. Because the intervention does not provide continuous chemoprophylaxis, some continued exposure should maintain naturally acquired immunity. Another concern about the widespread use of preventive treatment is the potential for drug resistance. While this concern is important, it should be weighed against the direct student health and potential indirect community benefits when evaluating the approach. Interventions should be designed to limit the potential for drug resistance by choosing drugs for prevention that differ from treatment drugs and proactively monitoring for drug resistance.

Our results join previous studies in supporting the need for implementation studies to measure the indirect impact of school-based treatment on community-level malaria transmission. Although RDTs will fail to detect some low-density infections, such as those observed in the dry season and in our lowest prevalence school, a screen-and-treat approach may substantially reduce the population of gametocytes in the community. Alternatively, providing preventive treatment to all students as intermittent preventive treatment, may have even greater impact. While higher density gametocyte-containing infections are more likely infectious, low density infections contribute to population-level transmission dynamics because they are common and, in our study population, less likely to be detected by $\mathrm{RDT}^{26}$. Future studies could also consider adding drugs targeting transmission, such as single-low dose primaquine, to enhance impact.

In summary, these results demonstrate the potential for school-based malaria treatment to substantially decrease $P$. falciparum gametocyte carriage and thus transmission in the surrounding community. In combination with disease- and vector-control interventions, this transmission-focused strategy may be critical to reduce the burden of malaria in areas where this disease has remained entrenched despite current control measures.

\section{Methods}

Study sites and design. School-based cohort studies and household-based cross-sectional surveys were conducted at the end of the rainy season (April-May) and during the dry season (September-October) of 2015. Two sites with consistently high $(>40 \%)$ parasite prevalence in school-age children-Maseya and Makhuwiraand two with lower, seasonally varied transmission (> two-fold seasonal prevalence difference) - Bvumbwe and Ngowe-were selected from 30 previously studied sites in southern Malawi (Figure S1) ${ }^{9}$. Long-lasting insecticide treated bed nets were distributed through a national campaign in 2012. Rapid diagnostic tests (RDTs) and treatment with artemether-lumefantrine were generally available in local government-operated health facilities.

School-based cohorts. Fifteen students per grade-level (grades 1-8) were sampled using a random number generator. Students were excluded if they: were less than 5 years old, were older than 15 years, had no parent/ guardian available to provide consent, would not attend school throughout the 6-week study, or had a known artemether-lumefantrine allergy. Students enrolled in the rainy season cohort were excluded from the dry season cohort. Guardians of sampled students were invited to information sessions and written informed consent was sought.

Enrolled students were interviewed at baseline (1-2 weeks after enrollment), one-, two-, and six-week visits about bed net use the night prior, current or recent illness, and antimalarial treatment. At baseline, a finger-prick blood sample was obtained for detection of $P$. falciparum by the same histidine-rich protein 2-based RDT used in the public sector (Paracheck Orchid Biomedical Systems, Goa, India or SD Bioline, Standard Diagnostics Inc., 
Suwon City, Republic of Korea) and hemoglobin was measured by portable photometer (Hemocue, Angelholm, Sweden). RDT sensitivity and specificity were $91.1 \%$ and $76.1 \%$, respectively, compared to microscopy. RDTpositive students received weight-based treatment course of artemether-lumefantrine (Novartis Pharma AG or Ajanta Pharma Ltd.). At baseline and all follow-up visits, finger-prick blood was obtained for molecular detection of any-stage parasites (filter paper dried blood spots) and gametocytes (whole blood in RNA preservative). At the final visit, parents were interviewed about their child's health during the study and students' health passports (individual portable medical records) reviewed to identify intercurrent fever or malaria treatment.

Household-based cross-sectional surveys. Concurrently cross-sectional surveys were conducted in 80 households in each school catchment area, including a cluster of 30 from ongoing surveillance studies (details in ${ }^{9}$ ) and 50 households newly selected based on closest Euclidian distance to the school. Households were visited within two weeks of the school cohort baseline visit. Questionnaires based on the Malaria Indicator Survey were administered and filter paper (Whatmann \#3) dried blood spots and whole blood in RNA preservative were obtained for polymerase chain reaction (PCR) testing, as previously described ${ }^{9,14}$. RDTs were not performed and no treatment was provided. School attendance in the last four weeks was asked for all participants $\geq$ five years. The majority of children started school at age 6 years, thus school-age age defined as six through 15 years old. Younger children were defined as 6 months through 5 years old, and adults as over 15 years old.

Plasmodium infection detection methods. At the school-based study baseline visit a histidine-rich protein 2-based RDTs were used per manufacturer instructions. For detection of any stage parasites on dried blood spots, real-time PCR was performed to detect the $P$. falciparum lactate dehydrogenase (PfLDH) gene, as described previously ${ }^{14}$. Assays were run in duplicate. If $P f L D H$ positive in either run, quantitative reverse transcription PCR was performed on $50 \mu \mathrm{l}$ whole blood preserved in $250 \mu \mathrm{l}$ RNAprotect Cell Reagent (Qiagen Inc. Valencia, CA, USA) to measure expression of the mature gametocyte marker $P f s 25$ (details provided in Supplemental Information) ${ }^{33}$.

Infection and treatment definitions. Plasmodium falciparum infection was defined as positive PfLDH PCR results on at least one run. Participants without evidence of $P f$ infection by PfLDH PCR were considered gametocyte negative. RDT-positive students with negative PCRs were considered negative for active infection, but were included in the treatment group for analysis because they had received artemether-lumefantrine. Other malaria treatment during follow-up was defined as any treatment with an effective antimalarial reported during student follow-up interviews, during the parent interview, or in the student's health passport.

Statistical analysis. All analyses of the school-based population accounted for survey weights and school sampling strata (Supplementary Materials). Contribution to transmission was evaluated four ways: (1) gametocyte prevalence defined as the proportion of participants with any gametocytes; (2) gametocyte density; (3) total gametocyte burden calculated as the sum of participant gametocyte densities; and (4) prevalence of infections with $\geq 10$ gametocytes/microliter $(\mu \mathrm{l})$, as there is an increased likelihood of infectiousness above this gametocyte density ${ }^{26}$.

For school-based cohorts at baseline, logistic regression models were used to assess determinants of gametocyte prevalence and linear models (log10-transformed) were used to assess determinants of gametocyte density among infections with $>0$ gametocytes. We used logistic regression among students with gametocyte-containing infections to identify characteristics of students in whom RDTs failed to detect gametocyte-containing infections at baseline. Gametocyte density was included as a continuous variable after assessing linearity of the relationship with odds of RDT negativity. Because of baseline differences in transmission pattern and demographics, all analyses were adjusted for school. In all models, selection of relevant predictors was based on Wald tests through best subset regression. Effect in linear models was reported as percent difference in absolute gametocyte density and was calculated as $[\exp ($ coeff $)-1] \times 100$.

Differences in the prevalence of gametocytemia and gametocyte density over time between treated (RDT positive) and untreated students (RDT negative) were assessed in nested random intercept longitudinal models by testing an interaction term between treatment and indicator variables for visit. Based on these longitudinal models, predictions of gametocyte prevalence and density were obtained, marginal on random intercepts, and stratified by school, season, and treatment status (baseline RDT positive or negative), with other modeled predictors assumed constant.

To assess the contribution of school-age children to the overall community population of gametocytes, we compared the distribution of all gametocyte-containing infections, infections containing $\geq 10$ gametocytes/ $\mu \mathrm{l}$, and geometric mean gametocyte density through chi-squared tests and linear regression: (i) across age-groups, and (ii) between students in the school-cohort and school-age children in the community. This analysis was unweighted because sampling of community surveys was not stratified.

Predictive modeling. To evaluate the potential indirect impact of school-based preventive treatment on the community, we estimated the expected reduction in the number of gametocyte-containing infections, gametocyte burden, and the number of infections containing $\geq 10$ gametocytes/ $\mu \mathrm{l}$ in the communities surrounding schools if the screen-and-treat intervention had included all students. Briefly, we first calculated the number of children from the community likely to be in school from the age- and gender-weighted proportion of schoolage children that reported attending school in the past four weeks. To estimate the expected reduction in the numbers of gametocyte-containing infections and high-density gametocyte-containing infections in the communities surrounding schools, we used mixture of distributions and conditional probabilities. To estimate the 
impact of the intervention on the overall gametocyte burden, we first fit overdispersed Poisson models to the school-based cohort with gametocyte density and RDT positivity results at baseline, then predicted the overall gametocyte density changes following the intervention for the hypothetical population of school-going children from the community who would have had a malaria infection and been treated in the screen-and-treat intervention (RDT-positive at baseline) at each follow-up time point. We assumed the population prevalence of gametocyte-containing infections in adults and younger children would remain constant during the intervention period because they would not receive the intervention.

Analyses were conducted in Stata/SE version 15.1 (StataCorp, College Station, TX, USA) and R v3.5 (R Foundation for Statistical Computing, Vienna, Austria $)^{34}$. Weighted analyses used the survey module of Stata. Significance was set at alpha $=0.05$. Further details of the predictive modeling and statistical analysis are provided in the Supplemental Information.

Ethical considerations. Written informed consent was obtained from participants over the age of 18 and from parents/guardians for participants under the age of 18 for both the school cohorts and the household surveys. Assent was obtained minors 13-17 years old. Participants could withdraw at any time. The University of Malawi College of Medicine Research and Ethics Committee and the Institutional Review Board of the University of Maryland Baltimore approved this study. The study was performed in accordance with relevant guidelines and regulations from these oversight bodies.

Received: 18 January 2021; Accepted: 16 March 2021

Published online: 25 March 2021

\section{References}

1. World Health Organization. World Malaria Report 2019. Geneva. (2019). Licence: CC BY-NC-SA 3.0 IGO

2. WHO. Global technical strategy for malaria 2016-2030. World Health Organization (2015).

3. Rabinovich, R. N. et al. malERA: An updated research agenda for malaria elimination and eradication. PLOS Med. 14, e1002456 (2017).

4. Barry, A. et al. Increased gametocyte production and mosquito infectivity in chronic versus incident Plasmodium falciparum infections. medRxiv (2020). https://doi.org/10.1101/2020.04.08.20057927

5. Bousema, T., Okell, L., Felger, I. \& Drakeley, C. Asymptomatic malaria infections: Detectability, transmissibility and public health relevance. Nat. Rev. Microbiol. 12, 833-840 (2014).

6. malERA: An updated research agenda for characterising the reservoir and measuring transmission in malaria elimination and eradication. PLoS Med. 14, (2017).

7. Nankabirwa, J. et al. Malaria in school-age children in Africa: An increasingly important challenge. Trop. Med. Int. Health https:// doi.org/10.1111/tmi.12374 (2014).

8. Stone, W., Gonçalves, B. P., Bousema, T. \& Drakeley, C. Assessing the infectious reservoir of falciparum malaria: Past and future. Trends Parasitol. https://doi.org/10.1016/j.pt.2015.04.004 (2015).

9. Walldorf, J. A. et al. School-age children are a reservoir of malaria infection in Malawi. PLoS ONE 10, e0134061 (2015).

10. Nankabirwa, J. I. et al. Estimating malaria parasite prevalence from community surveys in Uganda: A comparison of microscopy, rapid diagnostic tests and polymerase chain reaction. Malar. J. 14, 528 (2015).

11. Zhou, Z. et al. Assessment of submicroscopic infections and gametocyte carriage of Plasmodium falciparum during peak malaria transmission season in a community-based cross-sectional survey in western Kenya, 2012. Malar. J. 15, 421 (2016).

12. Mwandagalirwa, M. K. et al. Individual and household characteristics of persons with Plasmodium falciparum malaria in sites with varying endemicities in Kinshasa Province, Democratic Republic of the Congo. Malar. J. 16, 1-12 (2017).

13. Pinchoff, J. et al. Individual and household level risk factors associated with malaria in Nchelenge District, a region with perennial transmission: A serial cross-sectional study from 2012 to 2015. PLoS ONE 11, e0156717 (2016).

14. Coalson, J. E. et al. High prevalence of Plasmodium falciparum gametocyte infections in school-age children using molecular detection: Patterns and predictors of risk from a cross-sectional study in southern Malawi. Malar J 15, 1-17 (2016).

15. Lin Ouédraogo, A. et al. Dynamics of the human infectious reservoir for malaria determined by mosquito feeding assays and ultrasensitive malaria diagnosis in Burkina Faso. J. Infect. Dis. 213, 90-99 (2015).

16. Noor, A. M., Kirui, V. C., Brooker, S. J. \& Snow, R. W. The use of insecticide treated nets by age: Implications for universal coverage in Africa. BMC Public Health 9, 369 (2009).

17. Buchwald, A. G. et al. Bed net use among school-aged children after a universal bed net campaign in Malawi. Malar. J. 15, 127 (2016).

18. Port, G. R. \& Boreham, P. F. The relationship of host size to feeding by mosquitoes of the Anopheles gambiae Giles complex (Diptera: Culicidae). Bull. Entomol. Res. 70, 133-144 (1980).

19. Gonçalves, B. P. et al. Examining the human infectious reservoir for Plasmodium falciparum malaria in areas of differing transmission intensity. Nat. Commun. 8, 1133 (2017).

20. Coalson, J. E. et al. Simulation models predict that school-age children are responsible for most human-to-mosquito Plasmodium falciparum transmission in southern Malawi. Malar J 17, 1-12 (2018).

21. Cissé, B. et al. Effectiveness of seasonal malaria chemoprevention in children under ten years of age in Senegal: a stepped-wedge cluster-randomised trial. PLoS Med. 13, e1002175 (2016).

22. Cohee, L. M. et al. Preventive malaria treatment among school-age children in sub-Saharan Africa: A systematic review and metaanalyses. Lancet Glob. Heal. (2020, in press).

23. Clarke, S. E. et al. Impact of a malaria intervention package in schools on Plasmodium infection, anaemia and cognitive function in schoolchildren in Mali: A pragmatic cluster-randomised trial. BMJ Glob. Heal. 2, e000182 (2017).

24. Stuckey, E. M. et al. Modeling the cost effectiveness of malaria control interventions in the highlands of Western Kenya. PLoS ONE 9, e107700 (2014).

25. Staedke, S. G. et al. Assessment of community-level effects of intermittent preventive treatment for malaria in schoolchildren in Jinja, Uganda (START-IPT trial): A cluster-randomised trial. Lancet Glob. Heal. 6, e668-e679 (2018).

26. Bradley, J. et al. Predicting the likelihood and intensity of mosquito infection from sex specific Plasmodium falciparum gametocyte density. Elife 7, e34463 (2018). 
27. Uniting to Combat Neglected Tropical Diseases. Impact dashboard for preventive chemotherapy (PC) diseases. (2019). https:// unitingtocombatntds.org/impact-dashboards/pc-diseases-dashboard/. (2020)

28. Muirhead-Thompson, R. Distribution of anopheline mosquito bites among different age groups. Br. Med. J. 1, 1114 (1951).

29. Greenwood, B. M. et al. Mortality and morbidity from malaria after stopping malaria chemoprophylaxis. Trans. R. Soc. Trop. Med. Hyg. 89, 629-633 (1995).

30. Bradley-Moore, A. M. et al. Malaria chemoprophylaxis with chloroquine in young Nigerian children. I. Its effect on mortality, morbidity and the prevalence of malaria. Ann. Trop. Med. Parasitol. 79, 549-562 (1985).

31. Dicko, A. et al. Impact of intermittent preventive treatment with sulphadoxine-pyrimethamine targeting the transmission season on the incidence of clinical malaria in children in Mali. Malar. J. 7, 123 (2008).

32. Macete, E. et al. Intermittent preventive treatment for malaria control administered at the time of routine vaccinations in mozambican infants: a randomized, placebo-controlled trial. J. Infect. Dis. 194, 276-285 (2006).

33. Real time RT-PCR for presence and quantification of Plasmodium gametocytes from peripheral blood samples in RNAprotect. https://www.protocols.io/view/real-time-rt-pcr-for-presence-and-quantification-o-bh2sj8ee (2020).

34. R Development Core Team. R: A language and environment for statistical computing. (R Foundation for Statistical Computing, 2020).

\section{Acknowledgements}

We appreciate valuable input on the analysis and crafting of the manuscript from Drs. Kristin Stafford, Sania Amr, and Andrea Buchwald. We thank Heidi Fancher and the administrative team at the Malaria Alert Center for their support in executing the study. We are grateful for the hard work and dedication of the field team, study nurses, teachers, school administrators who made the study possible. Most importantly, we thank the participants for their commitment and patience.

\section{Author contributions}

Conceived of the study concept and design: L.M.C., C.V., M.L.W., M.K.L. Data collection and management: L.M.C., J.E.C., M.C., A.Ng., A.B., T.E.T., D.P.M., M.K.L. Laboratory analysis: A.Ny., K.B.S. Performed data analysis: L.M.C., C.V. Wrote the first draft of the manuscript: L.M.C., C.V., M.K.L. Agree with manuscript results and conclusions: L.M.C., C.V., J.E.C., A.Ny., M.C., A.Ng., A.B., K.B.S., M.L.W., T.E.T., D.P.M., M.K.L.

\section{Funding}

U.S. National Institutes of Health U19AI089683 (TET), K24AI114996 (MKL); and K23AI135076, Thrasher Research Fund Early Career Award, and Burroughs Wellcome Fund/American Society of Tropical Medicine and Hygiene Postdoctoral Fellowship in Tropical Infectious Diseases (LMC). Role of the funding source: The funders of the study had no role in study design, data collection, data analysis, data interpretation, or writing the report. The corresponding author had full access to all data and took final responsibility for the decision to submit for publication.

\section{Competing interests}

The authors declare no competing interests.

\section{Additional information}

Supplementary Information The online version contains supplementary material available at https://doi.org/ 10.1038/s41598-021-86450-5.

Correspondence and requests for materials should be addressed to L.M.C.

Reprints and permissions information is available at www.nature.com/reprints.

Publisher's note Springer Nature remains neutral with regard to jurisdictional claims in published maps and institutional affiliations.

(c) (i) Open Access This article is licensed under a Creative Commons Attribution 4.0 International License, which permits use, sharing, adaptation, distribution and reproduction in any medium or format, as long as you give appropriate credit to the original author(s) and the source, provide a link to the Creative Commons licence, and indicate if changes were made. The images or other third party material in this article are included in the article's Creative Commons licence, unless indicated otherwise in a credit line to the material. If material is not included in the article's Creative Commons licence and your intended use is not permitted by statutory regulation or exceeds the permitted use, you will need to obtain permission directly from the copyright holder. To view a copy of this licence, visit http://creativecommons.org/licenses/by/4.0/.

(c) The Author(s) 2021 